\title{
Endovascular Treatment in Patients with Cerebral Artery Occlusion of Three Different Etiologies
}

\author{
Dongwhane Lee, ${ }^{\mathrm{a}}$ Deok Hee Lee, ${ }^{\mathrm{b}}$ Dae Chul Suh, ${ }^{\mathrm{b}}$ Bum Joon Kim, ${ }^{\mathrm{c}}$ Sun U. Kwon, ${ }^{\mathrm{a}}$ Hyuk Sung Kwon, ${ }^{\mathrm{d}}$ \\ Ji-Sung Lee, ${ }^{\mathrm{e}}$ Jong S. Kim ${ }^{\mathrm{a}}$ \\ ${ }^{a}$ Department of Neurology, Asan Medical Center, University of Ulsan College of Medicine, Seoul, Korea \\ ${ }^{b}$ Department of Radiology, Asan Medical Center, University of Ulsan College of Medicine, Seoul, Korea \\ 'Department of Neurology, Kyung Hee University School of Medicine, Seoul, Korea \\ ${ }^{d}$ Department of Neurology, Hanyang University Guri Hospital, Hanyang University College of Medicine, Guri, Korea

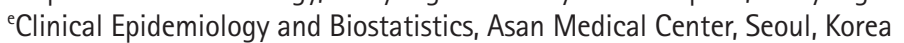

Background and Purpose The outcome of endovascular treatment (EVT) may differ depending on the etiology of arterial occlusion. This study aimed to assess the differences in EVT outcomes in patients with intracranial arterial steno-occlusion (ICAS-0), artery-to-artery embolism (AT-0), and cardiac embolism (CA-0).

Methods We retrospectively analyzed 330 patients with ischemic stroke who underwent EVT between January 2012 and August 2017. Patients were classified according to the etiology. The clinical data, EVT-related factors, and clinical outcomes were compared. The modified Rankin Scale (mRS) score at 3 months, determined using ordinal logistic regression (shift analysis), was the primary outcome.

Results CA-O ( $n=149)$ was the most common etiology, followed by ICAS- $0(n=63)$ and AT- $0(n=49)$. Age, initial National Institutes of Health Stroke Scale (NIHSS) score, and rate of hemorrhagic transformation were significantly higher in patients with CA- 0 compared to AT- 0 and ICAS- 0 . The time from onset-to-recanalization was the shortest in the CA-O (356.0 minutes) groups, followed by the AT-0 (847.0 minutes) and ICAS-0 (944.0 minutes) groups. The rates of successful recanalization, mRS distribution, and favorable outcomes at 3 months (mRS 0-2; CA-0, 36.9\%, AT-0, 53.1\%; and ICAS-0, 41.3\%) did not differ among the three groups. Baseline NIHSS score (odds ratio, 0.87; 95\% confidence interval, 0.83 to 0.91 ) could independently predict a favorable shift in mRS distribution.

Conclusions The functional outcomes of ICAS- 0 and AT- 0 were similar to those of CA-O, despite the delay in symptom onset-to-recanalization, suggesting that the therapeutic time window may be extended in these patients.

Keywords Stroke; Thrombectomy; Endovascular recanalization
Correspondence: Jong S. Kim Department of Neurology, Asan Medical Center, University of Ulsan College of Medicine, 88 Olympic-ro 43-gil, Songpa-gu, Seoul 05505, Korea

Tel: +82-2-3010-3440

Fax: +82-2-474-4691

E-mail: jongskim@amc.seoul.kr

Received: September 10, 2019

Revised: March 9, 2020

Accepted: April 29, 2020

\section{Introduction}

Endovascular treatment (EVT) has become the standard therapeutic modality for patients with acute ischemic stroke (AIS). ${ }^{1-4}$ However, the outcome of EVT may depend on the etiology of vascular occlusion. ${ }^{5}$ Although some studies have compared the outcome of EVT in patients with occlusion associated with intracranial arterial stenosis (ICAS-0) and embolic occlusion, more studies are needed to elucidate their differences. More importantly, none of these studies considered occlusion caused 
by artery-to-artery embolism (AT-0) and occlusion caused by cardiac embolism (CA-0) separately. ${ }^{5-16}$ The clinical outcomes of AT- 0 and CA-O may differ, because of the difference in the clot characteristics and collateral patterns of the two conditions. ${ }^{17}$ The present study evaluated the characteristics and outcomes of EVT in three different groups of patients with AIS, i.e., AT-0, CA-0, and ICAS-0.

\section{Methods}

\section{Study population}

Patients who presented with AIS caused by AT- 0 , CA-O, and ICAS-O, and underwent EVT between January 2012 and August 2017 at Asan Medical Center, Seoul, Korea, were consecutively enrolled. We excluded patients (1) with other (e.g., arterial dissection, moyamoya disease, cancer-related stroke, or vasculitis) or unknown etiologies (negative etiology or incomplete study), (2) with two or more potential causes of stroke (e.g., CA-O and ICAS-0, or AT-0 and ICAS-0), (3) who could not undergo EVT due to technical reasons (e.g., marked tortuosity), and (4) whose 90-day modified Rankin Scale (mRS) score could not be obtained. All clinical information, including baseline National Institutes of Health Stroke Scale (NIHSS) scores, was recorded. Patients were classified according to the Trial of ORG 10172 in Acute Stroke Treatment (TOAST) criteria. ${ }^{18}$

ICAS-0 was defined as follows: (1) residual stenosis $>70 \%$ in the target artery after thrombectomy, (2) moderate stenosis with flow and perfusion impairment on angiography or an evident tendency toward reocclusion, and (3) no source of embolism (e.g., significant proximal arterial stenosis or cardiac disease)..$^{19,20}$ The degree of stenosis was measured according to the WarfarinAspirin Symptomatic Intracranial Disease criteria. ${ }^{20}$ AT- 0 was defined as follows: (1) $>50 \%$ stenosis in the relevant proximal artery (internal carotid artery, common carotid artery [up to the levels of $\mathrm{C} 1]$, vertebral artery [V1-3 segment], common carotid artery, or severe atherosclerosis of the aorta) associated with relevant, distal artery occlusion; and (2) absence of embolism of cardiac origin. ${ }^{18}$ CA-0 was defined when patients had cardiac disease with medium or high-risk of stroke, as defined by the TOAST classification. ${ }^{18}$

This study was approved by the Institutional Review Board of the Asan Medical Center (IRB number: 2019-0110), which waived the requirement for written informed consent due to its retrospective nature.

\section{Endovascular treatment}

All patients with AIS who visited the emergency department were administered recombinant tissue plasminogen activator (rtPA) $(0.9 \mathrm{mg} / \mathrm{kg})$ intravenously within 3 hours of symptom onset, until December 2012 and within 4.5 hours after January 2013, if computed tomography (CT) showed no signs of brain hemorrhage. Patients underwent multi-modal (diffusionweighted, perfusion-weighted, fluid-attenuated inversion recovery [FLAIR], and gradient-echo) magnetic resonance imaging (MRI) and magnetic resonance angiography (MRA) while receiving rtPA. The clot sign on gradient-echo MRI was defined as a hypointense signal that exceeded the contralateral vessel diameter. ${ }^{21}$ EVT was performed in patients with large vessel occlusion and diffusion-perfusion mismatch or diffusion-clinical mismatch. Patients who were unable to undergo magnetic resonance examination for any reason were evaluated using CT perfusion scanning and $\mathrm{CT}$ angiography. Patients who could not be treated with rtPA due to late arrival underwent EVT alone, based on the MRI and MRA results. The decision to perform EVT was made through discussions between the attending neurologist and the neurointerventionist. EVT was generally performed $<24$ hours after symptom onset but was occasionally performed $\geq 24$ hours in patients who strictly met the criteria. If the exact onset time was unknown, it was defined as the point in time when the patient was lastly confirmed to be normal. EVTs were performed by one of two experienced neurointerventionists (DCS or DHL) as described elsewhere. ${ }^{22}$ Endovascular procedures including direct stenting and/or balloon angioplasty, mechanical disruption, direct aspiration, and a stent retriever were employed appropriately at the discretion of the neurointerventionist. Moreover, the decision to treat proximal severe stenosis/occlusion first or distal embolism first in patients with AT-O depended on the operator.

\section{Evaluation of clinical and angiographic outcomes} The patients were treated at the neurological intensive care unit after the procedure. Patients underwent follow-up CT/CT angiography or MRI/MRA at 24 to 48 hours to assess the presence of possible brain hemorrhage and confirm the maintenance of recanalization. The respective times from symptom onset to femoral puncture and reperfusion, from femoral puncture to reperfusion, and the total intervention time were measured. Reperfusion time was defined as the time to achieve a modified thrombolysis in cerebral infarction (mTICl) score $\geq 2 b^{23}$ Successful reperfusion was defined as an $\mathrm{mTICl}$ score of $2 b$ or 3 . The onset to recanalization time and puncture to recanalization time were calculated in patients in whom achieved recanalization was successful. The presence of cerebral hemorrhage on imaging scans obtained approximately 24 to 48 hours after the procedure was noted. Intracerebral hemorrhage $(\mathrm{ICH})$ was classified according to the method described by the European Cooperative Acute Stroke Study I trial. ${ }^{24}$ The clinical outcomes were assessed by stroke neurologists 
using the mRS 3 months after the stroke. mRS evaluation was performed via telephone by a trained nurse if the patient could not visit the hospital. The distribution of mRS scores (shift analysis) at 90 days was the primary outcome. ${ }^{25}$ The rates of change in the mRS score (0-2) at 3 months and the presence/absence of cerebral hemorrhage were the secondary outcomes.

Although no study has compared the outcomes of CA-O, AT0 , and ICAS-0, we performed a literature search (English language) on papers that compared ICAS- 0 and embolic occlusion in PubMed, Ovid, and MEDLINE published before October 2019 using MeSH terms or keywords including stroke, intracranial atherosclerosis, endovascular, mechanical thrombectomy, thrombectomy, stenosis, and stenting. Moreover, we found studies that were excluded by the search through a manual search. We included all these papers and tried to summarize the outcomes and predicting factors.

\section{Statistical analysis}

The categorical variables were compared using Pearson's chisquare or Fisher's exact test, wherever appropriate. Continuous demographic, clinical, and radiological variables were compared using one-way analysis of variance or the Kruskal-Wallis test. The 3-month outcomes of the three groups (shift analysis) were compared by calculating the common adjusted odds ratios (aORs) and 95\% confidence intervals (Cls). Variables with a $P<0.1$ on univariate analysis were included as candidate variables in multivariate analysis. Ordinal logistic regression was performed to evaluate factors that independently predicted outcomes after EVT. All $P$ values were two-sided and $P<0.05$ was statistically significance. Statistical analyses were performed using SPSS version 21.0 for Windows (IBM Corp., Armonk, NY, USA) and SAS version 9.4 (SAS Institute, Cary, NC, USA).

\section{Results}

\section{Patient characteristics}

We enrolled 330 AIS patients who underwent EVT between January 2012 and July 2017. Of these, 54 patients were eliminated based on the exclusion criteria (Figure 1). The remaining patients were divided into the embolic group $(n=198)$ and the ICAS-0 $(n=63)$ group. The remaining patients were divided into three groups: AT-0 ( $n=49), C A-0(n=149)$, and ICAS-0 $(n=63)$, after excluding patients with concomitant embolism and ICAS $(n=12)$, and patients with unavailable 3-month mRS scores $(n=15)$.

Patients in the CA- 0 group were older, with a female predominance, and had more severe neurological deficits than those in the ICAS- 0 and AT- 0 groups (Table 1). The frequency of diabetes and a current smoking habit were higher in the AT- 0 than that in the CA- 0 and ICAS- 0 groups. There were no significant differences in the location of occlusion, except that internal carotid arterial occlusion was more frequent in the AT-0 group. Clot signs were observed more frequently in the CA- 0 than those in the AT- 0 and ICAS- 0 groups.

\section{Procedural and clinical outcomes}

The time from symptom onset-to-initial recanalization was the longest in the ICAS-0 group (944 minutes; interquartile range [IQR], 511 to 2,155), followed by the AT-0 (847 minutes; IOR,

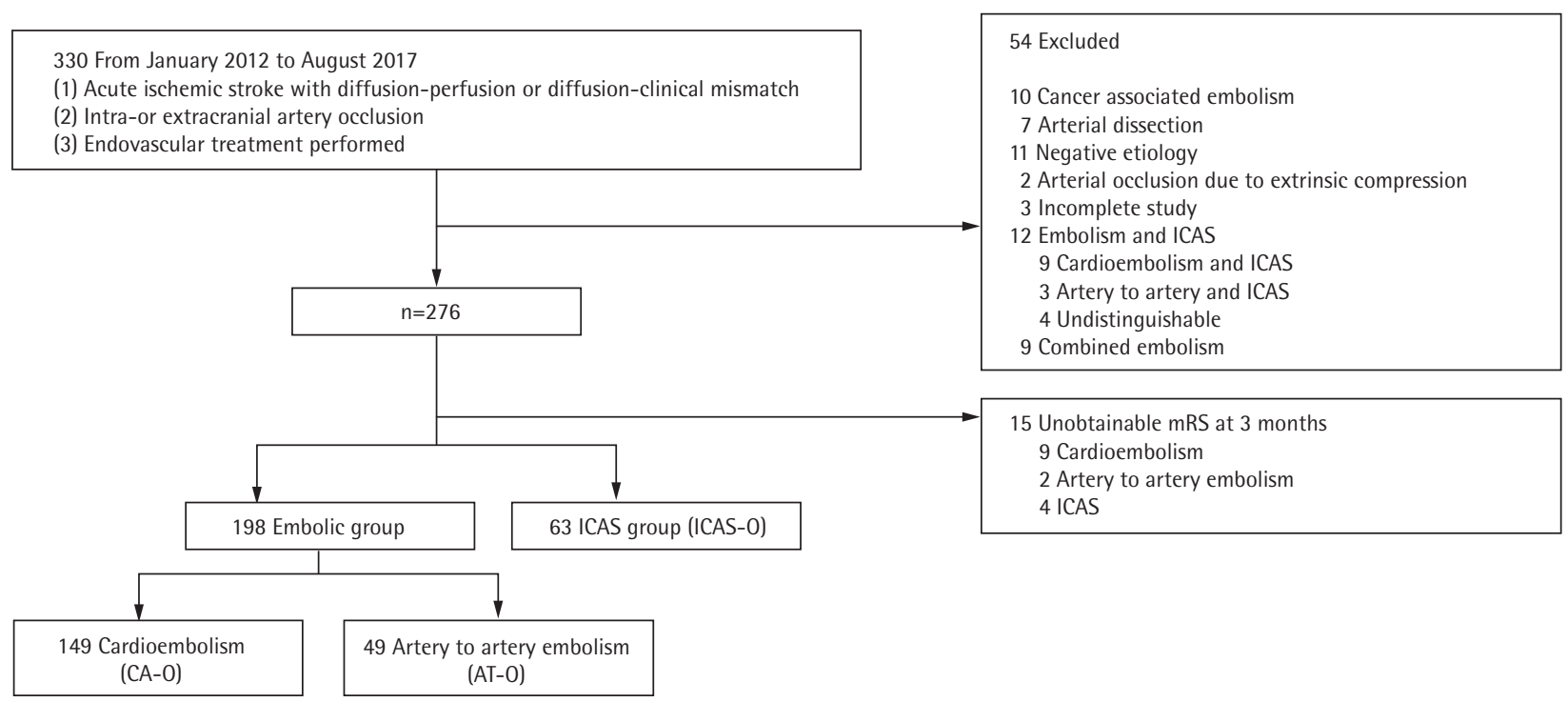

Figure 1. Flowchart of the patient selection process. ICAS, intracranial arterial steno; mRS, modified Rankin Scale; ICAS-0, intracranial arterial steno-occlusion; CA-O, occlusion caused by cardioembolism; AT-0, occlusion caused by artery-to-artery embolism. 
Table 1. Baseline characteristics of patients with ischemic stroke caused by three different etiologies

\begin{tabular}{|c|c|c|c|c|}
\hline Characteristic & CA-0 $(n=149)$ & AT-0 $(n=49)$ & ICAS-0 $(n=63)$ & $P$ \\
\hline Age (yr) & $69.7 \pm 10.9$ & $66.1 \pm 10.2$ & $66.2 \pm 10.7$ & 0.034 \\
\hline Male sex & $73(49.0)$ & 43 (87.8) & $50(79.4)$ & $<0.001$ \\
\hline Initial NIHSS & $14(11-17.5)$ & $10(7.5-13.5)$ & $11(8-15)$ & $<0.001$ \\
\hline Hypertension & $91(61.1)$ & $29(59.2)$ & 45 (71.4) & 0.301 \\
\hline Diabetes & $32(21.5)$ & $20(40.8)$ & $18(28.6)$ & 0.026 \\
\hline Hyperlipidemia & $34(22.8)$ & $14(28.6)$ & $20(31.7)$ & $<0.384$ \\
\hline Cigarette smoking & $36(28.8)$ & $12(50.0)$ & $7(15.2)$ & 0.010 \\
\hline Previous history of stroke & $29(19.5)$ & $14(28.6)$ & $14(22.2)$ & 0.424 \\
\hline Atrial fibrillation & 122 (81.9) & $0(0)$ & $0(0)$ & $<0.001$ \\
\hline \multicolumn{5}{|l|}{ Laboratory findings } \\
\hline Hemoglobin $(\mathrm{g} / \mathrm{dL})$ & $12.9 \pm 1.9$ & $14.0 \pm 1.9$ & $14.5 \pm 1.9$ & $<0.001$ \\
\hline Total cholesterol (mg/dL) & $168.2 \pm 44.0$ & $169.0 \pm 43.7$ & $181.9 \pm 37.6$ & 0.089 \\
\hline LDL-C (mg/dL) & $107.6 \pm 35.8$ & $104.0 \pm 40.8$ & $117.0 \pm 34.2$ & 0.132 \\
\hline C-reactive protein $(\mathrm{mg} / \mathrm{dL})$ & $1.1 \pm 2.5$ & $0.6 \pm 1.8$ & $0.9 \pm 1.9$ & 0.256 \\
\hline \multicolumn{5}{|l|}{ Occlusion site } \\
\hline Middle cerebral artery & $84(56.5)$ & $20(40.8)$ & $34(54.0)$ & 0.165 \\
\hline M1 & $71(47.7)$ & $17(34.7)$ & $28(44.4)$ & 0.291 \\
\hline M2 & $13(8.7)$ & $4(8.2)$ & $7(11.1)$ & 0.836 \\
\hline Anterior cerebral artery & $4(2.7)$ & $1(2.0)$ & $0(0)$ & 0.483 \\
\hline Posterior circulation arteries & $23(15.4)$ & $14(28.6)$ & $15(23.8)$ & 0.084 \\
\hline Internal carotid artery & $48(32.2)$ & $27(55.1)$ & $14(22.2)$ & 0.001 \\
\hline Clot sign & $67(45.0)$ & $11(22.4)$ & 25 (39.7) & 0.018 \\
\hline Treatment with intravenous tPA & $54(36.2)$ & $12(24.5)$ & $14(22.2)$ & 0.078 \\
\hline \multicolumn{5}{|l|}{ Time variables (min) } \\
\hline Onset to admission & $133.0(43.0-327.5)$ & $247.0(111.5-754.0)$ & $397(125-733.0)$ & $<0.001$ \\
\hline Onset to groin puncture & $282.0(190.0-522.5)$ & $773.0(282.0-1995.0)$ & $871(425.0-2,063.0)$ & $<0.001$ \\
\hline Onset to recanalization & $356.0(252.0-584.0)$ & $847(371.0-2,065.0)$ & $944(511.0-2,155.0)$ & $<0.001^{*}$ \\
\hline Puncture to recanalization & $55.0(37.0-77.0)$ & $62.0(52.0-89.5)$ & $78.0(55.0-107.0)$ & $<0.001^{+}$ \\
\hline Total intervention & $70.0(50.5-90.5)$ & $80.0(65.0-100.5)$ & $88.0(70.0-118.0)$ & $<0.001$ \\
\hline \multicolumn{5}{|l|}{ Modalities of EVT } \\
\hline Stent retriever & 119 (79.9) & $30(61.2)$ & $29(46.0)$ & $<0.001$ \\
\hline Suction thrombectomy & $61(40.9)$ & $23(46.9)$ & $7(11.1)$ & $<0.001$ \\
\hline Angioplasty & $6(4.0)$ & $37(75.5)$ & 45 (71.4) & $<0.001$ \\
\hline Stent insertion & $8(5.4)$ & $29(59.2)$ & $33(52.4)$ & $<0.001^{\dagger}$ \\
\hline Use of tirofiban & $2(1.3)$ & $4(8.2)$ & $8(12.7)$ & 0.001 \\
\hline Instant re-thrombosis & $1(0.7)$ & $4(8.2)$ & $9(14.3)$ & $<0.001$ \\
\hline $\mathrm{mTICl}$ & & & & 0.033 \\
\hline mTICl 0-1 & $10(6.8)$ & $3(6.1)$ & $1(1.6)$ & \\
\hline $\mathrm{mTICl} 2 \mathrm{a}$ & $6(4.0)$ & $1(2.0)$ & $4(6.3)$ & \\
\hline $\mathrm{mTICl} 2 \mathrm{~b}$ & $69(46.3)$ & $21(42.9)$ & 15 (23.8) & \\
\hline $\mathrm{mTICl} 3$ & $64(43.0)$ & $24(45.8)$ & $43(68.3)$ & \\
\hline Successful recanalization (mTICl 2b-3) & $136(91.3)$ & 45 (91.8) & $58(92.1)$ & $>0.999$ \\
\hline Recanalization in follow-up images & $127(94.1)$ & $37(90.2)$ & $47(81.0)$ & 0.020 \\
\hline $\mathrm{ICH}$ & $66(44.9)$ & $14(31.1)$ & $14(22.6)$ & 0.006 \\
\hline
\end{tabular}


Table 1. Continued

\begin{tabular}{|c|c|c|c|c|}
\hline Characteristic & CA-O $(n=149)$ & AT-0 $(n=49)$ & ICAS-0 $(n=63)$ & $P$ \\
\hline Type of ICH & & & & 0.462 \\
\hline Hemorrhagic infarction, 1 & $21(31.3)$ & $8(57.1)$ & $6(42.9)$ & \\
\hline Hemorrhagic infarction, 2 & $23(34.3)$ & $4(28.6)$ & $5(35.7)$ & \\
\hline Parenchymal hematoma, 1 & $10(14.9)$ & $2(14.3)$ & $2(14.3)$ & \\
\hline Parenchymal hematoma, 2 & 13 (19.4) & $0(0)$ & $1(7.1)$ & \\
\hline Subarachnoid hemorrhage & $3(2.0)$ & $0(0)$ & $1(1.7)$ & $>0.999$ \\
\hline Any cerebral hemorrhage & 69 (46.9) & $14(31.1)$ & $14(23.3)$ & 0.004 \\
\hline $\mathrm{mRS} 0-2$ at $3 \mathrm{mo}$ & $55(36.9)$ & $26(53.1)$ & $26(41.3)$ & 0.142 \\
\hline Mortality at $3 \mathrm{mo}$ & $13(8.7)$ & $2(4.1)$ & $6(9.5)$ & 0.552 \\
\hline
\end{tabular}

Values are presented as mean \pm standard deviation, number (\%), or median (interquartile range). The three groups were compared using Pearson chi-square tests, one-way analysis of variance (ANOVA) or the Kruskal-Wallis test, whichever was appropriate.

CA-O, occlusion caused by cardioembolism; AT-0, occlusion caused by artery-to-artery embolism; ICAS- 0 , intracranial arterial steno-occlusion; NIHSS, National Institutes of Health Stroke Scale; LDL-C, low-density lipoprotein cholesterol; tPA, tissue plasminogen activator; EVT, endovascular treatment; mTICl, modified thrombolysis in cerebral infarction; ICH, intracerebral hemorrhage; mRS, modified Rankin Scale.

${ }^{*},+$ Onset-to-recanalization time and puncture-to-recanalization time were calculated in patients in whom successful recanalization was achieved; ${ }^{*}$ Stent insertion refers to intracranial stenting in patients with ICAS- 0 or CA-O and extracranial (proximal) stenting in patients with AT-0.

Score on the Modified Rankin Scale at 3 months

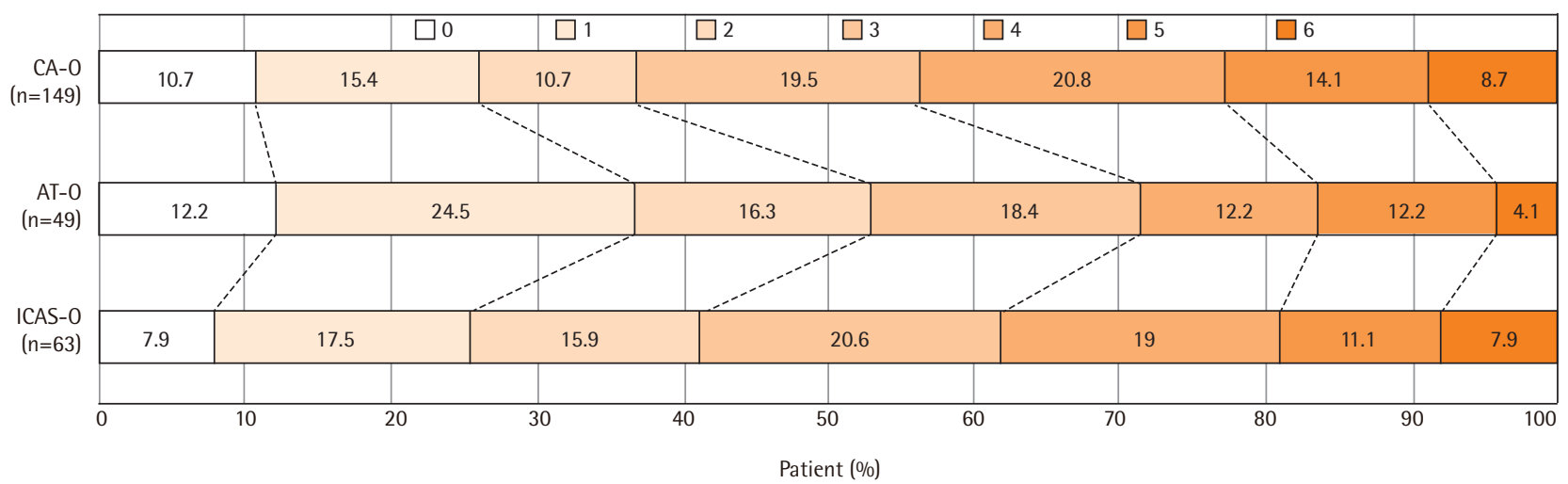

Figure 2. Distribution of the modified Rankin Scale scores 3 months after endovascular treatment among patients with occlusion caused by cardioembolism (CA-0), occlusion caused by artery-to-artery embolism (AT-0), and intracranial arterial steno-occlusion (ICAS-0). No significant difference was observed among these three groups.

371 to 2,065) and CA-0 (356 minutes; IQR, 252 to 584) groups $(P<0.001)$. The time from femoral puncture-to-recanalization $(P<0.001)$ and the total intervention time $(P<0.001)$ were also significantly longer in the ICAS- 0 and AT- 0 groups than that in the CA-O group. Balloon angioplasty and stenting were performed significantly more frequently in the ICAS-0 and AT-0 groups than that in the CA-O group. The rate of successful recanalization did not differ among the three groups, but the maintenance of recanalization at follow-up was significantly less frequent in the ICAS- 0 group than that in the other groups $(P<0.020)$. The rates of cerebral hemorrhage $(P=0.004)$ and $\mathrm{ICH}$ $(P=0.006)$ were significantly higher in the $\mathrm{CA}-0$ group than those in the AT-0 and ICAS-0 groups (Table 1).
The distribution of the mRS scores obtained 3 months after stroke ( $P=0.169$ using the Cochran-Mantel-Haenszel test) (Figure 2$)$ and the proportion of patients with $m R S$ scores of $0-2$ at 3 months ( $P=0.142)$ did not differ among the three groups. Ordinal logistic regression showed that baseline NIHSS score (aOR, $0.87 ; 95 \% \mathrm{Cl}, 0.83$ to $0.91 ; P<0.001)$ was the only significant independent predictor of poor outcomes (Table 2). The 3-month $m R S$ scores were similar in patients who underwent $\mathrm{EVT} \geq 24$ and $<24$ hours after symptom onset, as well as in patients who underwent EVT $\geq 6$ and $<6$ hours after symptom onset (Table 3 ). The comparison of the outcomes after EVT according to the patient recruitment period is described in Supplementary Table 1. 


\section{Discussion}

We examined the outcome of EVT based on three different mechanisms underlying vascular occlusion. CA-O was the most common etiology, accounting for $57 \%$ of patients, followed by ICAS-0 (24\%) and AT-0 (19\%). Although previous studies have attempted to determine the differences between patients with

Table 2. Ordinal logistic regression analysis of factors associated with the 3-month modified Rankin Scale scores in patients with acute ischemic stroke

\begin{tabular}{lcc}
\hline Variable & Common OR (95\% CI) & $P$ \\
\hline Age (/10 yr) & $0.89(0.72-1.10)$ & 0.286 \\
Male sex & $0.73(0.43-1.23)$ & 0.235 \\
Diabetes & $1.12(0.68-1.85)$ & 0.657 \\
Total cholesterol (/10 mg/dL) & $1.05(1.00-1.11)$ & 0.066 \\
Current smoking & $1.24(0.69-2.23)$ & 0.475 \\
Initial NIHSS & $0.87(0.83-0.91)$ & $<0.001$ \\
ICA occlusion & $1.10(0.68-1.79)$ & 0.692 \\
Onset to puncture time $(/ 1 \mathrm{hr})$ & $1.00(0.99-1.01)$ & 0.910 \\
Angioplasty & $1.27(0.66-2.43)$ & 0.473 \\
Type of etiologies & & \\
AT-0 (reference) & 1 & 0.182 \\
CA-0 & $0.62(0.31-1.25)$ & 0.939 \\
ICAS-0 & $1.03(0.48-2.20)$ & \\
Type of etiologies & & 0.182 \\
ICAS-0 (reference) & $1.60(0.80-3.21)$ & 0.150 \\
AT-0 & $1.65(0.83-3.28)$ & \\
CA-0 & & \\
\hline
\end{tabular}

Common ORs were calculated using ordinal logistic regression models indicating a favorable shift in modified Rankin Scale score distribution. $\mathrm{OR}$, odds ratio; $\mathrm{Cl}$, confidence interval; NIHSS, National Institutes of Health Stroke Scale; ICA, internal carotid artery; AT-O, occlusion caused by artery-to-artery embolism; CA- 0 , occlusion caused by cardioembolism; ICAS-0, intracranial arterial steno-occlusion.
ICAS-0 and 'embolic' occlusion, ${ }^{5-9,12,13,26}$ they did not distinguish between AT-O and CA-O in the 'embolic' group.

We found that duration of symptom onset-to-door time, puncture time, recanalization time, and the total intervention time were the longest in patients with ICAS- 0 , followed by those with AT-O and CA-0. Differences in the duration between symptom onset-to-door time were probably associated with the different characteristics of stroke among the three groups: $\mathrm{CA}-\mathrm{O}$ is characterized by sudden arterial occlusion without sufficient collaterals. In contrast, arterial occlusion associated with chronic atherosclerotic diseases is characterized by relatively mild or fluctuating symptoms because of better developed collaterals, although some of the latter patients show a progressive worsening of symptoms. ${ }^{27,28}$ We assumed that the longer onset-to-groin puncture time in patients with ICAS- 0 and AT- 0 may be attributed to the fact that EVT was often performed only after the observation of worsening or fluctuation in symptoms after the early stages, when the symptoms were mild. Despite the longer onset to EVT and intervention time, the outcomes were similar among the three groups.

Table 4 summarizes the results of studies comparing EVT outcomes in patients with ICAS- 0 and embolism. ${ }^{5-16,19,29,30}$ One study reported that patients with ICAS-O have better prognosis than those with embolism, ${ }^{7}$ while another study reported the opposite result in patients who received EVT in the vertebrobasilar territory. ${ }^{9}$ However, most studies did not report differences in 3 month clinical outcome after EVT between patients with ICAS-0 and those with embolism, ${ }^{7,10-16,26}$ which has also been highlighted by a recent meta-analysis. ${ }^{31}$ These studies reported that $16 \%$ to $89 \%$ of patients with ICAS-0 required stenting, and many were treated with tirofiban. ${ }^{6,7,12,26}$ One study found that rescue stenting, tirofiban treatment, or repeated thrombectomy resulted in favorable outcomes in $45 \%$ of pa-

Table 3. Comparison of patients according to different symptom onset-to-endovascular therapy times

\begin{tabular}{|c|c|c|c|c|c|c|c|c|}
\hline \multirow[b]{2}{*}{ Variable } & \multicolumn{4}{|c|}{ Etiologies } & \multirow{2}{*}{$\begin{array}{l}\text { Baseline } \\
\text { NIHSS }\end{array}$} & \multirow[b]{2}{*}{$P$} & \multirow{2}{*}{$\begin{array}{c}\text { Good outcome } \\
\text { (mRS 0-2 at } 3 \text { mo) }\end{array}$} & \multirow[b]{2}{*}{$P$} \\
\hline & Cardioembolism & $\begin{array}{c}\text { Artery to artery } \\
\text { embolism }\end{array}$ & $\begin{array}{l}\text { Intracranial arterial } \\
\text { steno-occlusion }\end{array}$ & $P$ & & & & \\
\hline $6 \mathrm{hr}$ & & & & $<0.001$ & & $<0.001$ & & 0.796 \\
\hline$<6 \mathrm{hr}(\mathrm{n}=98)$ & 75 (76.5) & $12(12.2)$ & 11 (11.2) & & $13(10-17.3)$ & & 39 (39.8) & \\
\hline$\geq 6 \mathrm{hr}(\mathrm{n}=163)$ & $74(45.4)$ & $37(22.7)$ & $52(31.9)$ & & $11(9-16)$ & & $68(41.7)$ & \\
\hline $24 \mathrm{hr}$ & & & & $<0.001$ & & $<0.001$ & & 0.629 \\
\hline$<24 \mathrm{hr}(\mathrm{n}=213)$ & $139(65.3)$ & 33 (15.5) & 41 (19.2) & & $8(9-16)$ & & $89(41.8)$ & \\
\hline$\geq 24 \mathrm{hr}(\mathrm{n}=48)$ & $10(20.8)$ & 16 (33.3) & $22(45.8)$ & & $10(7-13)$ & & 18 (38.5) & \\
\hline
\end{tabular}

Values are presented as number (\%) or median (interquartile range).

NIHSS, National Institutes of Health Stroke Scale; mRS, modified Rankin Scale. 


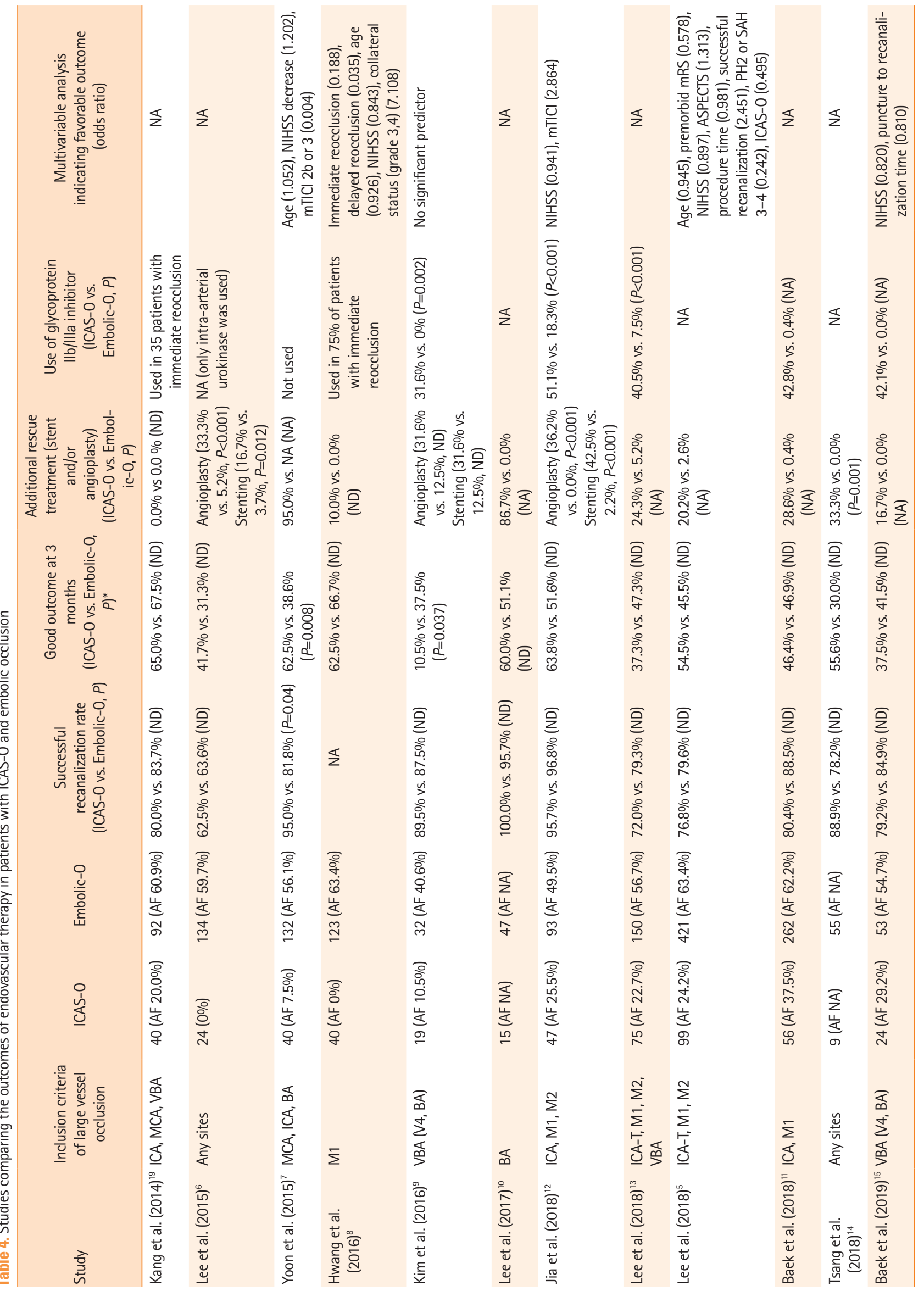




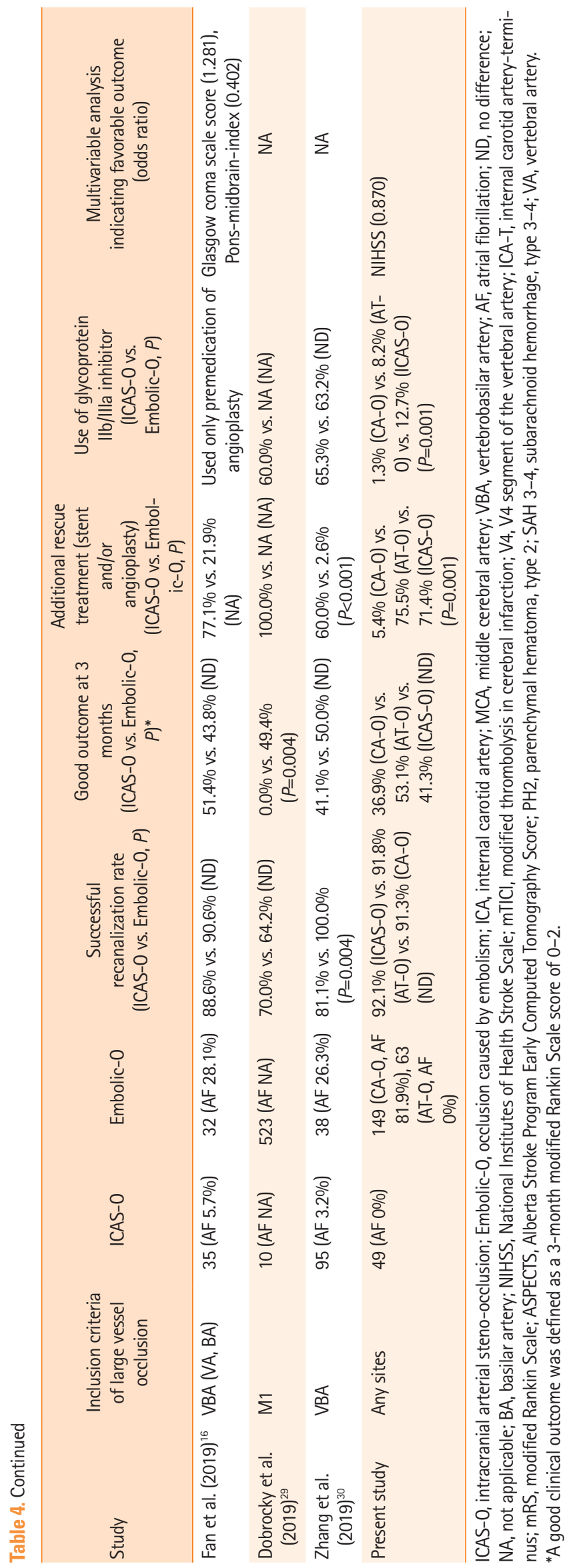

tients with ICAS-0 who underwent instant vascular reocclusion. ${ }^{8}$ Thus, although residual stenosis and reocclusion may be predictors of unfavorable outcomes, appropriate rescue treatment may have yielded similar outcomes in patients with ICAS- 0 and those with CA- $0 .{ }^{8}$

Our study is unique, since we classified patients with AT-0 into a separate category, which allowed us to observe the characteristics and EVT outcome in this group of patients. We found that the intervention time was longer in patients with AT-O than that in patients with CA-O (although it was shorter than that in ICAS-0), mainly due to the additional need to treat proximal arterial steno-occlusion. ${ }^{32,33}$ More than half of the patients with AT- 0 required stent insertion and $75 \%$ required angioplasty for the proximal artery. However, the rate of procedural complications was low overall, and post-stent rethrombosis was treated with tirofiban. Patients with AT-0 were younger, predominantly men, and had relatively less severe initial symptoms than patients with CA-O (similar to ICAS-0), perhaps due to relatively well-developed collaterals. Therefore, although AT-0 has been categorized as 'embolism' in previous studies, its characteristics differ from those of CA-O. The demographic and clinical features of AT-0 resembled those of ICAS-O, while the delay in the EVT procedure ranked between patients with CA-O and ICAS-O (Table 1). Regarding the technical issue in the treatment of tandem lesions, some interventionists favor the proximal (extracranial) lesion first approach, ${ }_{1}^{34}$ while others (including those at our center) favor the distal (intracranial) lesion first approach. A recent meta-analysis found no significant difference between the distal and proximal lesion first techniques (favorable outcome at 3 months: $53 \%$ vs. $49 \%, P=0.58$ )..$^{35}$ The outcome in our patients (favorable outcome, $53 \%$ ) was identical with the result of this meta-analysis.

Thus, patients with ICAS- 0 and AT- 0 are associated with more difficult and longer procedure times, and a higher frequency of re-occlusions compared to patients with CA-0. On the other hand, they tend to be younger and have less severe initial clinical symptoms, which are associated with better developed collaterals, while CA-O is associated with more severe initial symptoms and higher rates of hemorrhagic transformation. ${ }^{28,31}$ Moreover, adequate rescue treatment including angioplasty, stenting, and administration of glycoprotein IIb/IIla inhibitors has been used in patients with ICAS-0 and AT-0. Thus, the functional outcomes were overall similar among patients with AT-0, ICAS-0, and CA-0.,

Our study included patients who underwent EVT $\geq 24$ hours after symptom onset if certain conditions were met (e.g., diffusion-perfusion or diffusion-clinical mismatch). We found that the delayed treatment was significantly more often performed 
in patients with ICAS- 0 and AT- 0 than that in patients with in CA-O (Table 3) and that the functional outcomes in these patients did not differ from those in patients who underwent EVT $<24$ hours. Thus, EVT $\geq 24$ hours may still be beneficial if patients are carefully selected, especially in those with ICAS-0 and AT- $0 .{ }^{36}$ There were also no significant differences in functional outcomes between patients who underwent EVT $<12$ or $\geq 12$ hours and $<6$ or $\geq 6$ hours after symptom onset (Table 3 ).

Finally, when the study period was dichotomized (before and after 2015), we found that the 3-month clinical outcome after EVT was worse in the later stage than that in the early stage, although the onset-to-door time and onset-to-recanalization time were shortened (Supplementary Table 1). This may be attributed to the fact that the participants enrolled in the later stage were older, had more severe initial neurological deficits, and tended to have atrial fibrillation, reflecting in part the rapid increase in the aging population and atrial fibrillation in South Korea. Alternatively, candidates for EVT might have been selected more cautiously before the publication of five pivotal positive trials in 2015, while cases with greater severity were included afterwards. Unfortunately, we were unable to provide a satisfactory explanation with this retrospective study that enrolled patients who underwent EVT only. We also found that the functional outcomes did not differ among patients with CA-O, AT-0, and ICAS- 0 in the early or later stage (data not shown).

The merit of the present study was that the etiologies of stroke were clearly categorized based on the appropriate imaging and etiological examination. Moreover, this approach is closer to real-world scenarios: we did not exclude patients whose symptom onset was $>24$ hours, and included patients with AIS with anterior and posterior circulation involvement. Our study also has some limitations. First, this study was performed at a single center with an Asian population, and the results may not be generalizable to other ethnic groups. Second, although we believe that the relatively mild symptoms in patients with ICAS- 0 and AT- 0 patients were due to adequate collateral flow, the degree of collateral vessel formation was not assessed objectively. Third, the exclusion of patients with missing 3-month mRS scores due to loss to follow-up may have introduced a selection bias. However, the mRS scores at discharge did not differ among the patients excluded from the three groups ( $P=0.139$, data not shown). Fourth, in the current study we defined AT-0 as proximal stenosis was greater than $50 \%$ and excluded patients with concomitant AT-O and ICAS0 . However, the possibility of AT- 0 cannot be completely excluded in patients with milder proximal artery stenosis.

Despite these limitations, our data showed that the 3-month EVT outcomes did not differ among patients with AT-O, ICAS-
$\mathrm{O}$, and $\mathrm{CA}-\mathrm{O}$, although the symptom onset-to-recanalization time was longer in the ICAS- 0 and AT- 0 groups than that in the CA- 0 group. These findings suggest that ICAS- 0 and AT- 0 , which are characterized by no history of cardiac disease, no clot signs, small lesions on diffusion-weighted imaging, and a fluctuating or progressive diseases course may have longer therapeutic time windows than previously considered. Further studies are needed to determine the characteristics of the patients who would still improve despite the delayed therapy and how long the therapeutic window can be extended. This issue may be more important in Asian countries, where ICAS is more prevalent. $^{37}$

\section{Supplementary materials}

Supplementary materials related to this article can be found online at https://doi.org/10.5853/jos.2019.02404.

\section{Disclosure}

The authors have no financial conflicts of interest.

\section{References}

1. Saver JL, Goyal M, van der Lugt A, Menon BK, Majoie CB, Dippel DW, et al. Time to treatment with endovascular thrombectomy and outcomes from ischemic stroke: a metaanalysis. JAMA 2016;316:1279-1288.

2. Albers GW, Marks MP, Kemp S, Christensen S, Tsai JP, OrtegaGutierrez S, et al. Thrombectomy for stroke at 6 to 16 hours with selection by perfusion imaging. N Engl J Med 2018;378: 708-718.

3. Nogueira RG, Jadhav AP, Haussen DC, Bonafe A, Budzik RF, Bhuva P, et al. Thrombectomy 6 to 24 hours after stroke with a mismatch between deficit and infarct. N Engl J Med 2018; 378:11-21.

4. Ko SB, Park HK, Kim BM, Heo JH, Rha JH, Kwon SU, et al. 2019 Update of the Korean clinical practice guidelines of stroke for endovascular recanalization therapy in patients with acute ischemic stroke. J Stroke 2019;21:231-240.

5. Lee JS, Lee SJ, Yoo JS, Hong JH, Kim CH, Kim YW, et al. Prognosis of acute intracranial atherosclerosis-related occlusion after endovascular treatment. J Stroke 2018;20:394-403.

6. Lee JS, Hong JM, Lee KS, Suh HI, Demchuk AM, Hwang YH, et al. Endovascular therapy of cerebral arterial occlusions: intracranial atherosclerosis versus embolism. J Stroke Cerebrovasc Dis 2015;24:2074-2080.

7. Yoon W, Kim SK, Park MS, Kim BC, Kang HK. Endovascular 
treatment and the outcomes of atherosclerotic intracranial stenosis in patients with hyperacute stroke. Neurosurgery 2015;76:680-686.

8. Hwang YH, Kim YW, Kang DH, Kim YS, Liebeskind DS. Impact of target arterial residual stenosis on outcome after endovascular revascularization. Stroke 2016;47:1850-1857.

9. Kim YW, Hong JM, Park DG, Choi JW, Kang DH, Kim YS, et al. Effect of intracranial atherosclerotic disease on endovascular treatment for patients with acute vertebrobasilar occlusion. AJNR Am J Neuroradiol 2016:37:2072-2078.

10. Lee YY, Yoon W, Kim SK, Baek BH, Kim GS, Kim JT, et al. Acute basilar artery occlusion: differences in characteristics and outcomes after endovascular therapy between patients with and without underlying severe atherosclerotic stenosis. AJNR Am J Neuroradiol 2017;38:1600-1604.

11. Baek JH, Kim BM, Heo JH, Kim DJ, Nam HS, Kim YD. Outcomes of endovascular treatment for acute intracranial atherosclerosis-related large vessel occlusion. Stroke 2018;49:2699-2705.

12. Jia B, Feng $L$, Liebeskind DS, Huo $X$, Gao F, Ma N, et al. Mechanical thrombectomy and rescue therapy for intracranial large artery occlusion with underlying atherosclerosis. J Neurointerv Surg 2018;10:746-750.

13. Lee JS, Lee SJ, Hong JM, Choi JW, Yoo J, Hong JH, et al. Solitaire thrombectomy for acute stroke due to intracranial atherosclerosis-related occlusion: ROSE ASSIST study. Front Neurol 2018;9:1064.

14. Tsang ACO, Lau KK, Tsang FCP, Tse MMY, Lee R, Lui WM. Severity of intracranial carotid artery calcification in intracranial atherosclerosis-related occlusion treated with endovascular thrombectomy. Clin Neurol Neurosurg 2018;174:214216.

15. Baek JH, Kim BM, Heo JH, Kim DJ, Nam HS, Kim YD. Endovascular and clinical outcomes of vertebrobasilar intracranial atherosclerosis-related large vessel occlusion. Front Neurol 2019;10:215.

16. Fan $Y$, Li Y, Zhang T, Li X, Yang J, Wang B, et al. Endovascular therapy for acute vertebrobasilar occlusion underlying atherosclerosis: a single institution experience. Clin Neurol Neurosurg 2019;176:78-82.

17. Boeckh-Behrens T, Schubert M, Förschler A, Prothmann S,

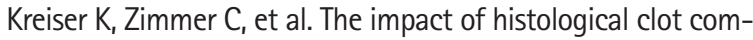
position in embolic stroke. Clin Neuroradiol 2016;26:189-197.

18. Adams HP Jr, Bendixen BH, Kappelle $\sqcup$, Biller J, Love BB, Gordon $\mathrm{DL}$, et al. Classification of subtype of acute ischemic stroke. Definitions for use in a multicenter clinical trial. TOAST. Trial of Org 10172 in Acute Stroke Treatment. Stroke 1993;24:35-41.

19. Kang DH, Kim YW, Hwang YH, Park SP, Kim YS, Baik SK. In- stant reocclusion following mechanical thrombectomy of in situ thromboocclusion and the role of low-dose intra-arterial tirofiban. Cerebrovasc Dis 2014;37:350-355.

20. Samuels OB, Joseph GJ, Lynn MJ, Smith HA, Chimowitz MI. A standardized method for measuring intracranial arterial stenosis. AJNR Am J Neuroradiol 2000;21:643-646.

21. Cho KH, Kim JS, Kwon SU, Cho AH, Kang DW. Significance of susceptibility vessel sign on $\mathrm{T} 2^{*}$-weighted gradient echo imaging for identification of stroke subtypes. Stroke 2005;36:23792383.

22. Lee $D$, Lee $D H$, Suh DC, Kwon HS, Jeong DE, Kim JG, et al. Intra-arterial thrombectomy for acute ischaemic stroke patients with active cancer. J Neurol 2019;266:2286-2293.

23. Zaidat 00 , Yoo AJ, Khatri P, Tomsick TA, von Kummer R, Saver $J \mathrm{~L}$, et al. Recommendations on angiographic revascularization grading standards for acute ischemic stroke: a consensus statement. Stroke 2013:44:2650-2663.

24. Fiorelli M, Bastianello S, von Kummer R, del Zoppo GJ, Larrue $V$, Lesaffre $E_{\text {, et al. Hemorrhagic transformation within } 36}$ hours of a cerebral infarct: relationships with early clinical deterioration and 3-month outcome in the European Cooperative Acute Stroke Study I (ECASS I) cohort. Stroke 1999;30:2280-2284.

25. Saver JL, Gornbein J. Treatment effects for which shift or binary analyses are advantageous in acute stroke trials. Neurology 2009;72:1310-1315.

26. Al Kasab S, Almadidy Z, Spiotta AM, Turk AS, Chaudry MI, Hungerford JP, et al. Endovascular treatment for AIS with underlying ICAD. J Neurointerv Surg 2017;9:948-951.

27. Rebello LC, Bouslama M, Haussen DC, Grossberg JA, Dehkharghani S, Anderson A, et al. Stroke etiology and collaterals: atheroembolic strokes have greater collateral recruitment than cardioembolic strokes. Eur J Neurol 2017;24:762767.

28. Lee JS, Hong JM, Kim JS. Diagnostic and therapeutic strategies for acute intracranial atherosclerosis-related occlusions. J Stroke 2017;19:143-151.

29. Dobrocky T, Kaesmacher J, Bellwald S, Piechowiak E, Mosimann PJ, Zibold F, et al. Stent-retriever thrombectomy and rescue treatment of $m 1$ occlusions due to underlying intracranial atherosclerotic stenosis: cohort analysis and review of the literature. Cardiovasc Intervent Radiol 2019;42:863872.

30. Zhang $X$, Luo G, Jia B, Mo D, Ma N, Gao F, et al. Differences in characteristics and outcomes after endovascular therapy: a single-center analysis of patients with vertebrobasilar occlusion due to underlying intracranial atherosclerosis disease and embolism. Interv Neuroradiol 2019;25:254-260. 
31. Tsang ACO, Orru E, Klostranec JM, Yang IH, Lau KK, Tsang FCP, et al. Thrombectomy outcomes of intracranial atherosclerosis-related occlusions. Stroke 2019;50:1460-1466.

32. Sivan-Hoffmann R, Gory B, Armoiry X, Goyal M, Riva R, Labeyrie $P E$, et al. Stent-retriever thrombectomy for acute anterior ischemic stroke with tandem occlusion: a systematic review and meta-analysis. Eur Radiol 2017;27:247-254.

33. Yoo AJ, Andersson T. Thrombectomy in acute ischemic stroke: challenges to procedural success. J Stroke 2017;19:121-130.

34. Rangel-Castilla L, Rajah GB, Shakir HJ, Shallwani H, Gandhi $\mathrm{S}$, Davies JM, et al. Management of acute ischemic stroke due to tandem occlusion: should endovascular recanalization of the extracranial or intracranial occlusive lesion be done first? Neurosurg Focus 2017;42:E16.

35. Wilson MP, Murad MH, Krings T, Pereira VM, O'Kelly C, Rempel J, et al. Management of tandem occlusions in acute ischemic stroke: intracranial versus extracranial first and extracranial stenting versus angioplasty alone: a systematic review and meta-analysis. J Neurointerv Surg 2018;10:721728.

36. Albers GW. Late window paradox. Stroke 2018;49:768-771.

37. Lü PH, Park JW, Park S, Kim JL, Lee DH, Kwon SU, et al. Intracranial stenting of subacute symptomatic atherosclerotic occlusion versus stenosis. Stroke 2011;42:3470-3476. 
Supplementary Table 1. Comparison of the outcomes in EVT according to the recruitment period

\begin{tabular}{|c|c|c|c|}
\hline Variable & Until December 31, 2014 ( $n=139)$ & From January 1, 2015 (n=122) & $P$ \\
\hline Age (yr) & $66.5 \pm 10.9$ & $70.1 \pm 10.5$ & 0.007 \\
\hline Male sex & $93(66.9)$ & $73(59.8)$ & 0.248 \\
\hline Initial NIHSS & $11(8-15)$ & $13(10-17)$ & 0.005 \\
\hline Hypertension & $89(64.0)$ & $76(62.3)$ & 0.798 \\
\hline Diabetes & $36(25.9)$ & $34(27.9)$ & 0.780 \\
\hline Hyperlipidemia & $45(32.4)$ & $23(18.9)$ & 0.016 \\
\hline Atrial fibrillation & $57(41.0)$ & $65(53.3)$ & 0.062 \\
\hline Etiologies & & & 0.146 \\
\hline Cardioembolism & $72(51.8)$ & $77(63.1)$ & \\
\hline Artery to artery embolism & $31(22.3)$ & $18(14.8)$ & \\
\hline Intracranial arterial steno-occlusion & $36(25.9)$ & $27(22.1)$ & \\
\hline Onset to door time & $201.0(66.0-516.0)$ & $170.0(49.0-477.5)$ & 0.030 \\
\hline Onset to recanalization time (min) & $579.0(331.0-1,464.0)$ & $392.0(253.0-847.3)$ & 0.023 \\
\hline \multicolumn{4}{|l|}{ Modalities of EVT } \\
\hline Stent retriever & $83(59.7)$ & 95 (77.9) & 0.002 \\
\hline Suction thrombectomy & $56(40.3)$ & $35(28.7)$ & 0.052 \\
\hline Angioplasty & $60(43.2)$ & $28(23.0)$ & 0.001 \\
\hline Stent insertion & 49 (35.3) & $21(17.2)$ & 0.001 \\
\hline Use of tirofiban & $8(5.8)$ & $6(4.9)$ & 0.791 \\
\hline Good outcome (mRS 0-2 at $3 \mathrm{mo}$ ) & 65 (46.8) & $42(34.4)$ & 0.045 \\
\hline
\end{tabular}

Values are presented as mean \pm standard deviation, number (\%), or median (interquartile range).

EVT, endovascular treatment; NIHSS, National Institutes of Health Stroke Scale; mRS, modified Rankin Scale. 